\title{
Nonlinear dispersion of avalanche upconversion
}

\author{
Q. Shu, H. Ni, and S. C. Rand \\ Division of Applied Physics, 1049 Randall Laboratory, University of Michigan, Ann Arbor, Michigan 48109-1120 \\ Received August 12, 1996 \\ Two-beam coupling measurements of an avalanche upconversion transition in concentrated Tm:LiYF 4 confirm \\ that, despite the resonant nature of the excited-state optical interaction, the induced response is strongly \\ dispersive. This surprising characteristic is shown to be a general feature of avalanche polarization, with an \\ off-resonant process dominating the resonant response. (c) 1997 Optical Society of America
}

In recent years simple implementations of two-beam coupling interactions ${ }^{1}$ have been introduced for precision characterization of nonlinear coefficients ${ }^{2}$ and for studying slow radiative and nonradiative decay processes within luminescent centers in solids. ${ }^{3}$ Here we report results of nondegenerate two-beam coupling (TBC) mediated by delocalized, cooperative dynamics of impurity atoms on avalanche upconversion transitions ${ }^{4}$ and use them to investigate an earlier indication from research by $\mathrm{Ni}^{5}$ that avalanche nonlinearities are primarily dispersive, in apparent disagreement with existing models of resonant nonlinear response. ${ }^{6}$ We confirm this general feature of avalanche nonlinearities that nonresonant contributions dominate the nonlinear polarization of this type of resonant interaction and point out several important applications.

Basic features of avalanche absorption were modeled by Kueny et al. ${ }^{7}$ and others. ${ }^{8}$ Those authors pictured the cross relaxation of one excited- and one ground-state ion, which resulted in two excitedintermediate-state ions, as a key step in the underlying nonlinear process leading to a chain reaction of excitation driven by excited-state absorption. $\mathrm{Ni}^{9}$ subsequently pointed out the essential role of migration in the process, and numerous examples of avalanche absorption have since been reported. ${ }^{10}$ Significant applications have emerged in the areas of avalanche upconversion lasers, ${ }^{11}$ amplifiers, ${ }^{12}$ and signal processing. ${ }^{13}$ However, there have been few fundamental studies of the nonlinear-optical response associated with the avalanche itself. Here we provide direct evidence that the real part of the nonlinear index is much larger than the imaginary part, exactly in resonance with the avalanche transition.

In conventional theories of nonlinear optics a single strong input field in resonance with an allowed transition generates a purely absorptive change in the response. On the other hand, at large detunings the response is predicted to be dispersive. In avalanche upconversion an interesting situation is encountered in which two transitions are involved, one exactly on resonance and one far off resonance, as indicated at the left in Fig. 1(a). Intuitively, in the absence of coupling between the atoms one expects the resonant interaction to dominate. Hence the nonlinear response should be absorptive in character.

For coupled atoms, though, the situation is greatly modified. Above avalanche threshold, cross relaxation provides a mechanism for heavily populating excited states even when the initial optical interaction involving the ground state is far off resonance. The populations in excited states and the value of the predicted threshold intensity show little or no dependence on the weak ground-state absorption.,14 However, models that omit the ground-state transition altogether predict only nonlinear properties associated with the excited-state transition, which is purely absorptive on resonance. Because the excited state involved in the off-resonant ground-state transition can nevertheless be significantly populated because of avalanche processes, the polarization associated with the ground-state optical interaction is unexpectedly strong even when the ground-state absorption is negligible.

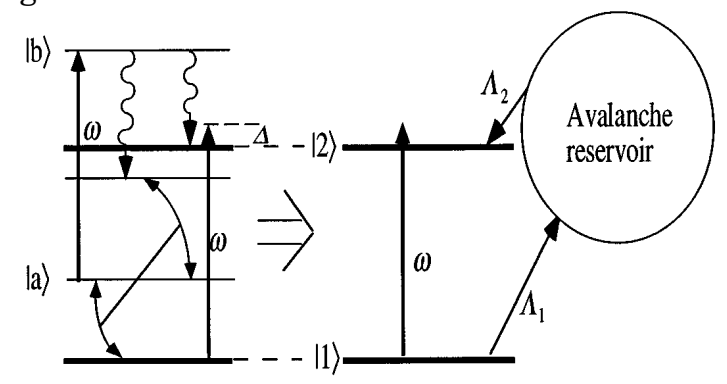

(a)

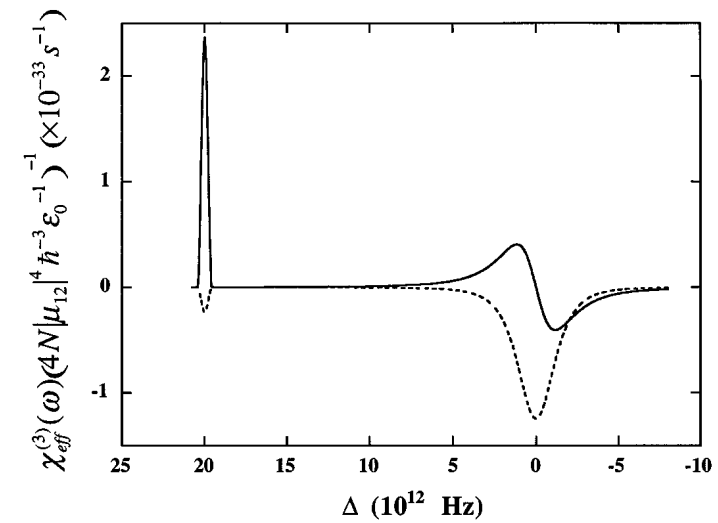

(b)

Fig. 1. (a) Reduction of a multilevel avalanche system to a two-level nonlinear system. Cross relaxation (curved arrows) and decay (wiggly arrows) account for incoherent pumping of excited states at large detunings from the ground-state transition. (b) Calculation of the susceptibility from Eq. (4). Solid dashed curves correspond to real and imaginary induced indices, respectively. The parameters used are $\Gamma_{21}=2 \times 10^{12} \mathrm{~s}^{-1}$ and $\gamma_{21}=2 \times 10^{8} \mathrm{~s}^{-1}$. 
To model this situation we replaced all but one excited state with an avalanche reservoir whose occupation is large but bears no particular phase relationship to the polarization developed on the off-resonant transition between the ground state and level $|2\rangle$. This quasi-two-level model is shown at the right in Fig. 1(a). Incoherent pumping and depletion of the reservoir are justified in the limit of large $\Delta$ by the spontaneous nature of the cross relaxation that populates state $|2\rangle$ and by spontaneous decay processes among excited levels. In steady state the equations of motion for the components of the density matrix are

$$
\begin{aligned}
& \dot{\rho}_{11}=\gamma_{21} \rho_{22}-\Omega v-\Lambda_{1}=0, \\
& \dot{\rho}_{22}=-\gamma_{21} \rho_{22}+\Omega v+\Lambda_{2}=0, \\
& \dot{\tilde{\rho}}_{21}=-\left(\Gamma_{21}-i \Delta\right) \tilde{\rho}_{21}+1 / 2 i \Omega\left(\rho_{22}-\rho_{11}\right)=0 .
\end{aligned}
$$

Here $\gamma_{21}$ and $\Gamma_{21}$ are the population decay and the optical dephasing rates, respectively. $\Delta=\omega-\omega_{21}$ is the detuning of the incident frequency $\omega$ from transition frequency $\omega_{21}$ between levels $|1\rangle$ and $|2\rangle, \Omega=$ $-2 \mu_{32} \tilde{E} / \hbar$ for fields of the form $E=\tilde{E} \exp (-i \omega t)+$ c.c., $\tilde{\rho}_{21}$ is the slowly varying amplitude of the offdiagonal element $\rho_{21}$ defined by $\rho_{21}=\tilde{\rho}_{21} \exp (-i \omega t)$, and $v$ is the imaginary part of $\tilde{\rho}_{12}=\tilde{\rho}_{21}{ }^{*}=u+$ $i v$. Closure requires that $\rho_{11}+\rho_{22}+\rho_{r}=1$, where reservoir population $\rho_{r}$ gives the total population density circulating among excited states other than $|2\rangle$. In steady state $\Lambda_{1}=\Lambda_{2}$, and we assume that this rate is proportional to occupation of the reservoir $\left(\Lambda_{2}=f \rho_{r}\right.$, where $f$ is the rate at which reservoir atoms decay to level $|2\rangle$ ).

The steady-state polarization $P(t)=N \operatorname{Tr}(\rho \mu)=$ $\tilde{P}(\omega) \exp (-i \omega t)+$ c.c. can be determined from Eqs. (1)(3) and used to calculate an effective susceptibility $\chi_{\text {eff }}$ according to $\tilde{P}(\omega)=\epsilon_{0} \chi_{\text {eff }}(\omega) \tilde{E}$. $\quad \chi_{\text {eff }}$ consists of the usual linear susceptibility $\chi^{(1)}(\omega)$ and a nonlinear, intensity-dependent part $\chi_{\text {eff }}^{(3)}(\omega)$. The nonlinear part is $^{14}$

$$
\begin{aligned}
& \chi_{\text {eff }}^{(3)}(\omega)=\left[\frac{4 N\left|\mu_{12}\right|^{4} \hbar^{-3} \epsilon_{0}{ }^{-1}\left(\Delta-i \Gamma_{21}\right) \Gamma_{21} / \gamma_{21}}{\left(\Delta^{2}+\Gamma_{21}{ }^{2}\right)\left(\Delta^{2}+\Gamma_{21}{ }^{2}+|\Omega|^{2} \Gamma_{21} / \gamma_{21}\right)}\right. \\
& \left.+\frac{4 N\left|\mu_{12}\right|^{4} \hbar^{-3} \epsilon_{0}^{-1}\left(\Delta-i \Gamma_{21}\right)\left(1+2 f / \gamma_{21}\right)}{\Delta^{2}+\Gamma_{21}{ }^{2}+|\Omega|^{2} \Gamma_{21} / \gamma_{21}}\left(\rho_{r} /|\Omega|^{2}\right)\right] .
\end{aligned}
$$

The first term in Eq. (4) corresponds to saturation of a conventional two-level system, whereas the second term arises from the incoherent pumping channel. The relative contributions of the two terms for various detunings $\Delta^{\prime}=\omega-\omega_{b a}$ of the light wave from the excited-state transition frequency vary considerably. For small detunings $\left[\Delta^{\prime}=\Delta-\left(\omega_{b a}-\omega_{21}\right) \approx 0\right]$ the first term may be much smaller than the second, because detuning from the ground-state transition under this condition $\left(\Delta=\omega_{b a}-\omega_{21}\right)$ is necessarily large for avalanche processes. In this case the nonlinear susceptibility is dominated by a real contribution that peaks at the excited-state resonance, as shown in Fig. 1(b) $\left(\Delta^{\prime}=0 \leftrightarrow \Delta=20\right)$. On the other hand, when $\Delta=0$ the first term can dominate, because $\rho_{r}$ tends to zero abruptly $\left(\Delta^{\prime} \neq 0\right)$ and the avalanche is extinguished. This situation generates broad features in the vicinity of the ground-state resonance $[\Delta=0$ in Fig. 1(b)], where the real nonlinear index passes through zero.

The total susceptibility is the sum of the off-resonant ground-state polarization from Eq. (4) and the resonant excited-state polarization that one obtains by ignoring the ground-state transition. The analytical results in Fig. 1(b) for optical susceptibility $\chi_{\text {eff }}^{(3)}(\omega)$ based on microscopic avalanche dynamics can be compared directly with measurable index changes observed by two-beam coupling. In the weak probe limit, experimental values for the Debye decay time $\tau$ that characterize the saturation process, as well as real and imaginary parts $n_{2}{ }^{\prime}$ and $n_{2}{ }^{\prime \prime}$ of the nonlinear refractive index $n_{2}$ [directly proportional to $\chi_{\text {eff }}^{(3)}(\omega)$ ], can be determined from the analysis of TBC spectra.

Experiments were performed with a pump-probe technique that we described previously, which makes use of a stabilized dye laser. ${ }^{3}$ Only one improvement over our earlier approach was implemented here. The output was split into two beams propagating with polarizations parallel to the optic axis and a third beam, which did not pass through the sample but provided an intensity reference for a differencing signal amplifier

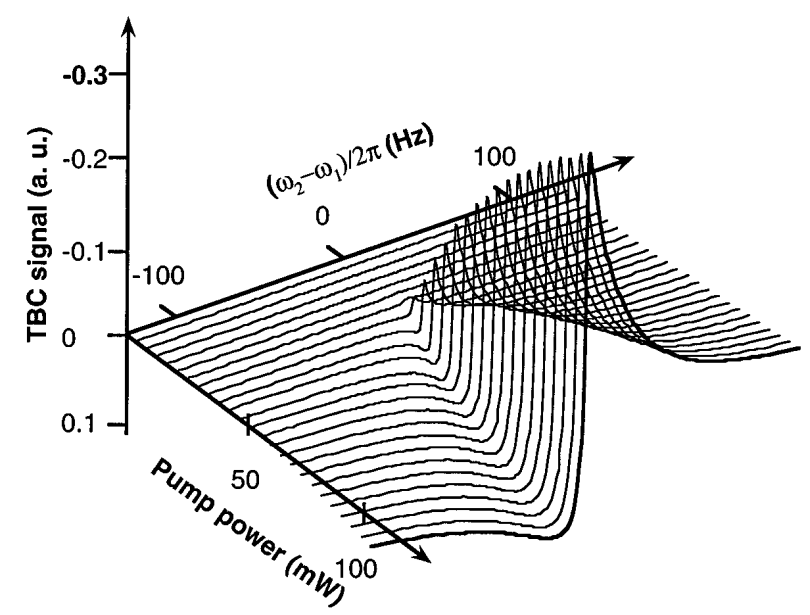

Fig. 2. Experimental two-beam coupling signals in $\mathrm{Tm}: \mathrm{LiYF}_{4}$ versus both pump-probe detuning and pump intensity.

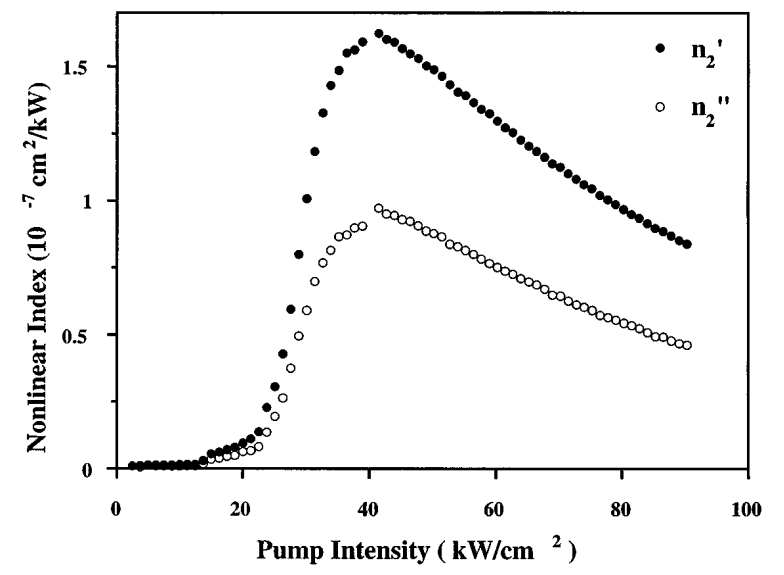

Fig. 3. Real $\left(n_{2}{ }^{\prime}\right)$ and imaginary $\left(n_{2}{ }^{\prime \prime}\right)$ nonlinear indices versus input intensity from data of Fig. 2. 


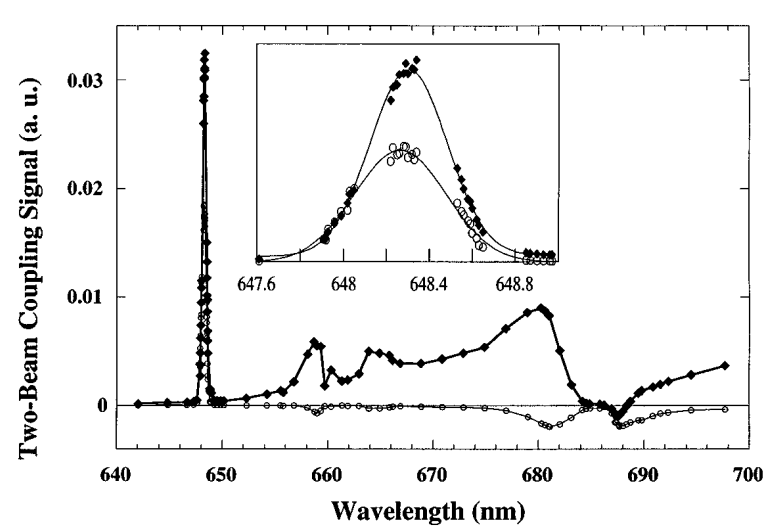

Fig. 4. Plot of the dispersive (filled diamonds) and absorptive (open circles) amplitudes of the TBC signals in $5 \% \mathrm{Tm}: \mathrm{LiYF}_{4}$ versus wavelength over a broad spectral region containing both ground- and excited-state transitions. Inset: Enlargement of the avalanche resonance region at $648 \mathrm{~nm}$. The slight skewing of the dispersive component line shape with respect to the absorptive component is due to the summation of ground- and excited-state polarization contributions. Data are uncorrected for sample absorption.

to reduce common mode intensity noise by $40 \mathrm{~dB}$. The samples were single crystals of Tm:LiYF 4 with concentrations of $1-12 \%$. The strongest avalanche response free of thermal grating contributions was obtained in a $1.5 \%$ sample $5 \mathrm{~mm}$ in length, which we used to measure the intensity dependence reported here. A 5\% sample $3 \mathrm{~mm}$ in length was used for all the widespectral-range measurements. Typical spectra recorded at various intensities are shown in Fig. 2. A striking feature of these data is the rapid rise in nonlinear response above a well-defined threshold intensity near $20 \mathrm{~kW} / \mathrm{cm}^{2}$. The shapes of individual spectral traces are well described by the superposition of absorptive and dispersive components for a single nonlinear resonance. ${ }^{3}$

The values of $n_{2}{ }^{\prime}$ and $n_{2}{ }^{\prime \prime}$ determined from TBC analysis are shown in Fig. 3. An abrupt rise in nonlinear response occurs just above the avalanche threshold at $20 \mathrm{~kW} / \mathrm{cm}^{2}$. Then, in the range $40-100 \mathrm{~kW} / \mathrm{cm}^{2}$, both the real and the imaginary parts diminish slowly because of saturation. ${ }^{14}$ The most interesting aspect of these data is that the real part $n_{2}{ }^{\prime}$ of the induced index is much larger than the imaginary part $n_{2}{ }^{\prime \prime}$. This behavior is contrary to predictions of resonant response in a two-level system.

These characteristics are nevertheless exactly these predicted by Eq. (4) when processes such as cross relaxation exist that permit incoherent pumping of an excited state in the presence of off-resonant excitation of ground-state centers. Figure 1(a) clearly shows the change in nonlinear response predicted by our model when incident light is tuned to a ground-state absorption resonance $(\Delta=0)$ or to an excited-state resonance $\left(\Delta^{\prime}=0 \leftrightarrow \Delta=20\right)$. These results are in excellent agreement with measurements (Fig. 4) that show that $n_{2}^{\prime}$ is zero at the $686.5 \mathrm{~nm}$ ground-state resonance but nonzero and dominant at the $648.2-\mathrm{nm}$ excited-state (avalanche) resonance.
Our results show that the avalanche nonlinearity observed in $\mathrm{Tm}: \mathrm{LiYF}_{4}$ at $648.2 \mathrm{~nm}$ gives rise to beam coupling with a sharp threshold and a dispersive character at room temperature. The real and the imaginary parts of the induced nonlinear index have large magnitudes, of the order of $10^{-14} \mathrm{~m}^{2} / \mathrm{W}$. The real index goes to zero as expected at ground-state resonant wavelengths but is large and dominant over the imaginary index at the excited-state (avalanche) resonance. This behavior is consistent with a simple density-matrix model, which reveals that the dispersive character of the nonlinear susceptibility is a general feature of any system that experiences significant pumping of an excited state at large detunings from any ground-state resonance. This dispersive nonlinearity could provide a new passive self-mode-locking mechanism for avalanche upconversion lasers and presents an intrinsic limitation to gain in avalanche upconversion fiber amplifiers.

H. Ni thanks the Barbour Scholarship Program for graduate support. The authors are indebted to N. Cockcroft and R. M. MacFarlane for samples.

\section{References}

1. M. A. Kramer, W. R. Tompkin, and R. W. Boyd, Phys. Rev. A 34, 2026 (1986); I. McMichael, P. Yeh, and P. Beckwith, Opt. Lett. 13, 500 (1988).

2. S. A. Boothroyd, J. Chrostowski, and M. S. O'Sullivan, J. Opt. Soc. Am. B 6, 766 (1989).

3. D. Redman, Q. Shu, A. Lenef, and S. C. Rand, Opt. Lett. 17, 175 (1992).

4. J. Chivian, W. Case, and D. Eden, Appl. Phys. Lett. 35, 124 (1979).

5. H. Ni, "Avalanche upconversion in $\mathrm{Tm}_{\mathrm{LiYF}}$, $\mathrm{TmYAlO}_{3}$ and Tm:YAG," Ph.D. dissertation (University of Michigan, Ann Arbor, Mich., 1994).

6. See, for example, Y. R. Shen, The Principles of Nonlinear Optics (Wiley, New York, 1984).

7. A. Kueny, W. E. Case, and M. E. Koch, J. Opt. Soc. Am. B 6, 639 (1989).

8. W. E. Case, M. E. Koch, and A. W. Kueny, J. Lumin. 45, 351 (1990); U. Oetliker, M. J. Riley, P. S. May, and H. U. Gudel, Coord. Chem. Rev. 111, 125 (1991); M. F. Joubert, S. Guy, and B. Jacquier, Phys. Rev. B 48, 10031 (1993).

9. H. Ni and S. C. Rand, Opt. Lett. 16, 1424 (1991).

10. T. Hebert, R. Wannemacher, R. M. Macfarlane, and W. Lenth, Appl. Phys. Lett. 60, 2592 (1992); Y. H. Chen and F. Auzel, Electron. Lett. 30, 1602 (1994); M. Pollnau, W. Luthy, and H. P. Weber, J. Appl. Phys. 77, 6128 (1995); R. Scheps, IEEE J. Quantum Electron. 31, 309 (1995).

11. T. Sandrock, E. Heumann, and G. Huber, in International Quantum Electronics Conference (Optical Society of America, Washington, D.C., 1996).

12. L. M. Yang, T. Sonsnowski, M. L. Stock, T. B. Norris, J. Squier, G. Mourou, M. L. Dennis, and I. Duling, Opt. Lett. 20, 1044 (1995).

13. H. Ni and S. C. Rand, Opt. Lett. 17, 1222 (1992).

14. Q. Shu, "Cooperative optical nonlinearities," Ph.D. dissertation (University of Michigan, Ann Arbor, Mich., 1996).

15. $\rho_{r}$ can be calculated by use of the standard four-level model, ${ }^{8}$ as we have done, or it can be treated as a free parameter. 